\section{Produção e avaliação do desempenho de painéis de partículas de madeira a partir de resíduos de podas de árvores urbanas}

Prodution and Evaluation of Performance of Particleboard Made of Urban Trees Pruning Residues

\section{RESUMO}

As espécies utilizadas na arborização urbana requerem periódicas podas para atender aos requisitos peculiares das essências e para garantir a segurança em relação às redes elétricas e de telefonia. $\mathrm{O}$ grande volume de material oriundo das podas tem potencial para, na forma de partículas, ser empregado na produção de painéis para emprego na confecção de móveis e para aplicação como componente de edificações. No presente trabalho, utilizaram-se resíduos de poda de árvores de Canelinha (Nectandra lanceolata), obtidos na cidade de São Carlos (SP). Foram produzidos painéis de madeira aglomerada, tendo como adesivo a resina poliuretana bicomponente à base de óleo de mamona, desenvolvida no Instituo de Química de São Carlos, da USP. Os parâmetros de processo foram: partículas de dimensão máxima 2,8 $\mathrm{mm}$; porcentagem de adesivo $16 \%$; temperatura de prensagem: $90^{\circ} \mathrm{C}$; tempo de prensagem: dez minutos. Os ensaios para avaliação do desempenho dos painéis produzidos foram feitos de acordo com as recomendações da NBR 14810:2006, da Associação Brasileira de Normas Técnicas. Os resultados evidenciaram a viabilidade técnica da referida produção, pois boa parte dos requisitos normativos foi atingida. Ajustes nos parâmetros de processo, principalmente no que tange à temperatura de prensagem, serão necessários para que os painéis atendam a todos os requisitos da NBR 14810:2006.

Palavras-chave: Painéis de partículas. Resíduos de poda de árvores urbanas. Avaliação de desempenho.

\section{ABSTRACT}

Species used in urban arborization require regular pruning to meet the unique essences requirements and to ensure safety in relation to electrical and telephonic nets. The large volume of material from pruning has the potential to be (in particulate shape) used in panel
EDUARDO CHAHUD Francisco Antonio Rocco Lahr, Karen Anéris Blecha, Maria Fátima do Nascimento e Marília da Silva B ERTOLIN I 
production for furniture manufacturing and for use as building components. In this study, we used tree pruning residues of Canelinha (Nectandra lanceolata), obtained in the city of São Carlos (SP). With this material, particleboards with the two-component castor oil based polyurethane resin (PUR) as adhesive were produced. PUR was developed in São Carlos Chemistry Institute, USP. The process parameters were: particle maximum dimension 2,8 mm; adhesive percentage $16 \%$; pressing temperature: $90^{\circ} \mathrm{C}$; pressing time: $10 \mathrm{~min}$. Tests for panel performance evaluation were carried out according to NBR 14810:2006 recommendations. Results demonstrated the technical feasibility of this production, once much of the regulatory requirements were achieved. Adjustments to process parameters, mainly regarding the pressing temperature, are needed in order to the panels reach all requirements of NBR 14810:2006.

Keywords: Particleboards. Residues of urban arborization pruning. Performance evaluation.

\section{INTRODUÇÃO}

As cidades brasileiras apresentam, em seu panorama urbano, uma quantidade relativamente significativa de árvores, em geral plantadas não somente com objetivos estéticos, mas também para amenizar as altas temperaturas que ocorrem no verão, em grande parte da área territorial habitada da nação.

A maioria das espécies empregadas requer periódica ação de poda de seus galhos, fundamentalmente para:

" Atender aos requisitos característicos das essências no que diz respeito ao seu desenvolvimento e longevidade;

" Garantir a segurança em relação aos fios de transmissão de energia elétrica (parcela elevada desta fiação ainda é aérea, fixada nos chamados "postes de eletrificação”).

A quantidade de resíduos gerada pela atividade da poda dos galhos das referidas árvores pode alcançar valores expressivos. Embora não haja registro bibliográfico nesse sentido, as observações feitas no decorrer deste trabalho permitem quantificar em aproximadamente $0,01 \mathrm{~m}^{3}$ por habitante, por ano. No tocante à cidade de São Carlos, considerando-se que sua população em 2010 alcance os 200 mil habitantes, o volume gerado poderia alcançar $2000 \mathrm{~m}^{3}$ ao ano, conforme Poleto et al. [13].

Estes resíduos, de maneira geral, são depositados nas áreas de descarte do lixo urbano e, embora não se constituam em graves ameaças de danos ambientais (em função da facilidade de decomposição), deveriam ser objeto de estudo para a verificação do potencial de seu emprego em aplicações que agregassem valor ao insumo e viessem a trazer benefícios à coletividade.

Nesse sentido foi desenvolvido o presente trabalho, que objetivou avaliar a viabilidade técnica do emprego dos resíduos de poda de árvores urbanas (mais especificamente na cidade de São Carlos) na produção de painéis de partículas, visando seu aproveitamento em móveis para uso nas próprias instalações do poder público municipal, em especial no mobiliário das escolas. 


\section{OBJETIVOS}

Conforme as informações apresentadas anteriormente, o objetivo do presente trabalho é produzir e avaliar, em laboratório, o desempenho de painéis de partículas de madeira produzidos com o material oriundo da poda de árvores urbanas. Optou-se pelo emprego, como adesivo, da resina poliuretana à base de óleo de mamona (bicomponente), desenvolvida por docentes do Instituto de Química de São Carlos (IQSC/USP).

\section{REVISÃO BIBLIOGRÁFICA}

Dadas as características do tema do trabalho, a bibliografia específica é praticamente inexistente no país. Nesse contexto, serão apresentados aspectos da produção brasileira de painéis particulados, com destaque para o aproveitamento de resíduos na produção deste tipo de painel (também chamado de madeira aglomerada) e para a resina poliuretana à base de óleo de mamona.

\section{Chapas de aglomerado}

A partir da madeira, é possível gerar novos produtos pela sua reconstituição utilizando métodos e processos adequados. Os painéis de madeira são produtos compostos de elementos como lâminas, sarrafos, partículas e fibras (cuja obtenção se dá a partir da redução da madeira sólida), reconstituídos por meio de ligação adesiva, conforme explicita Iwakiri [8].

O Brasil exibe ótimas condições para produzir os citados painéis, como mostrado no Gráfico 1, principalmente em função da possibilidade de cultivar, em larga escala, essências florestais de rápido crescimento, como as oriundas dos gêneros Pinus e Eucalyptus. Matéria prima renovável e com baixo consumo de energia para ser processada, a madeira é interessante insumo para muitos produtos [11].

Dentre os produtos à base de madeira, os painéis aglomerados vêm apresentando as maiores taxas de crescimento (Gráfico 2) em função da quantidade de produtos disponíveis e facilidades na aplicação para os mais variados fins.

O Ministério da Agricultura, Pecuária e Abastecimento prevê, no período de 2006 a 2012, investimento superior a US \$1,1 bilhão no setor. Como reflexo, a produção nacional de painéis de madeira, que em 2000 foi de 1,5 milhão de $\mathrm{m}^{3}$, pode alcançar 3 milhões em 2012. As empresas produtoras se localizam nas regiões Sul e Sudeste do Brasil e cerca de $80 \%$ dos painéis produzidos atendem à indústria moveleira. $\mathrm{O}$ restante tem sido empregado na construção civil e na indústria de embalagens.

Para a produção dos painéis particulados, a madeira é picada, transformada em partículas as quais são submetidas à secagem, até alcançar umidade da ordem de 8 a 10\%. O material, então, recebe uma resina e eventuais aditivos, seguindo para prensagem à temperatura próxima a $200^{\circ} \mathrm{C}[14]$. 
Gráfico 1 Produção brasileira de painéis de madeira. Fonte: BNDES.

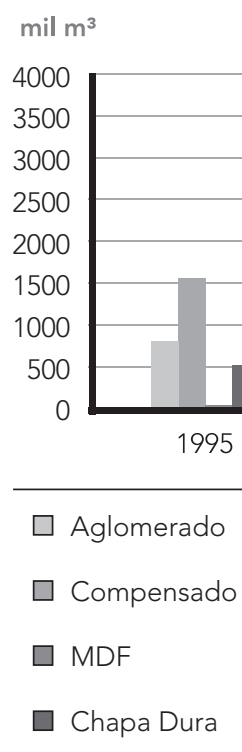

Gráfico 2 Produção mundial de painéis de madeira. Fonte: BNDES.

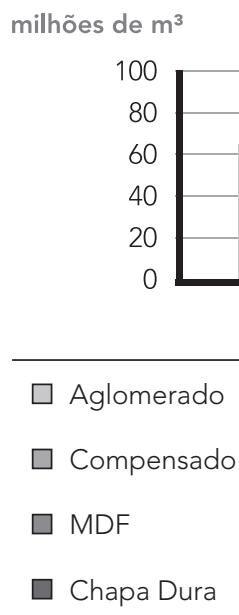

Aglomerado

$\square$ Compensado

MDF

Chapa Dura

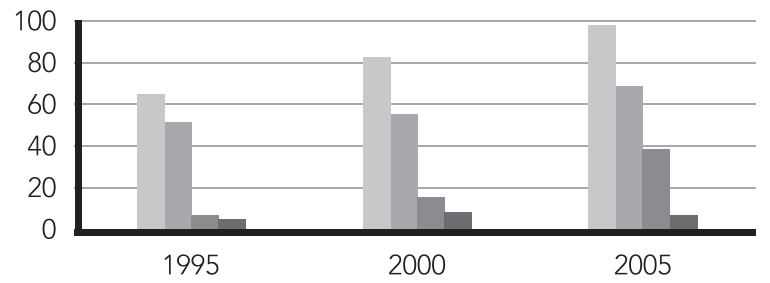

O princípio de fabricação faz com que o aglomerado apresente vantagens em relação a outros produtos compostos de madeira, e também à madeira serrada. Segundo Bodig e Jayne [5], Tomaselli [14], Nascimento [12], Iwakiri [8] e Dias [7], as vantagens são:

" Eliminação de efeitos da anisotropia;

" Resistência da chapa no sentido da largura e do comprimento é similar;

" Eliminação de fatores redutores da resistência como nós, inclinação da grã, lenhos 
juvenil e adulto, entre outros;

"Controle das propriedades físico-mecânicas por meio das variáveis de processo, como resina, geometria de partículas, grau de densificação;

" Menor exigência em termos de qualidade da matéria-prima;

" Menos mão de obra necessária e menor custo de produção.

\section{Aproveitamento de resíduos na produção de painéis de partículas}

Avaliações realizadas por Blanchet [3] sobre a confecção de chapas de madeira aglomerada com resíduo de costaneira de Abeto, concluíram ser viável o aproveitamento desses resíduos e que é tecnicamente possível a produção das chapas de madeira aglomerada conforme as exigências do American National Standard Institute (ANSI), para utilização em ambientes secos.

Ainda segundo Blanchet, as chapas foram confeccionadas em laboratório nas dimensões de $540 \times 560 \times 160 \mathrm{~mm}$, com resíduo seco em estufa na temperatura de $60^{\circ} \mathrm{C}$. Nas camadas superficiais foram utilizadas partículas de 0,02 a $2 \mathrm{~mm}$ e nas camadas internas foram distribuídas partículas de 2 a $6 \mathrm{~mm}$, obtidas por meio de moinho de martelo, e considerados três procedimentos experimentais:

1. Nas camadas superficiais foram utilizadas 10, 25 e $50 \%$ de partículas;

2. Para cada porcentagem de partícula distribuída nas superfícies das chapas foi adotado 12, 14 e 16\% de resina ureia-formaldeído (UF),;

3. Sendo $8 \%$ no interior das chapas; resultando numa combinação de $3 \times 9$ entre os tratamentos, o que definiu a confecção de 27 chapas de madeira aglomerada.

Os melhores resultados das propriedades mecânicas das chapas foram obtidos por meio dos tratamentos com $14 \%$ de resina UF nas camadas superficiais com $50 \%$ de partículas. Os experimentos apresentaram, entretanto, problemas de expansão linear devido à influência da umidade, o que determinou seu uso para ambientes secos.

Pesquisas sobre a produção de chapas de madeira aglomerada utilizando-se resíduos de costaneira de Eucalyptus saligna, Eucalyptus citriodora e Eucalyptus pilularis foram realizadas por Iwakiri [9], objetivando avaliar o comportamento das misturas dos resíduos dessas madeiras na produção de chapas aglomeradas com dois níveis de resina ureia-formaldeído, de acordo com a norma CS 236-66. As chapas foram produzidas em laboratório e os procedimentos experimentais se constituíram na utilização de partículas obtidas por meio de moinho de martelo com umidade de aproximadamente $3 \%$; dois níveis de resina ureia-formaldeído ( 8 e $12 \%$ na base peso seco das partículas); densidade nominal de $0,80 \mathrm{~g} / \mathrm{cm}^{3}$; ciclo de prensagem com temperatura de $140^{\circ} \mathrm{C}$, tempo de prensagem de oito minutos, pressão de prensagem de $40 \mathrm{kgf} / \mathrm{cm}^{2}$; sendo produzidos três chapas por tratamento, resultando na confecção de 21 chapas.

Ainda segundo Iwakiri, os resultados obtidos possibilitaram concluir que:

1. As chapas com $12 \%$ de resina UF apresentaram maior estabilidade dimensional, 
destacando-se o Eucalyptus saligna;

2. Não ocorreram diferenças significativas entre o módulo de elasticidade para os diferentes níveis de resina ( 8 e 12\%);

3. Foram determinados melhores resultados de módulo de ruptura para as chapas com $12 \%$ de resina UF confeccionadas com partículas de Eucalyptus saligna, e somente para esta espécie a maior quantidade de resina (12\%) influenciou positivamente no módulo de ruptura;

4. Os resultados obtidos para a ligação interna dos painéis, em todos os tratamentos, foram superiores aos referenciados pela norma CS 236-66;

5. Por fim, pode-se concluir que os resíduos de costaneira das espécies de eucalipto estudadas podem ser recomendados para produção de chapas de madeira aglomerada.

Avaliações no ciclo de prensagem realizadas por Albuquerque [1] mostraram a influência do incremento de água nas camadas superficiais do colchão de partículas e do incremento de resina e catalisador. $\mathrm{O}$ autor concluiu ser possível confeccionar chapas aglomeradas com partículas finas, sem necessidade de se utilizar mais que 10\% de ureia-formaldeído.

Para o caso das chapas com partículas grossas, observou-se que é necessário um tempo superior a quatro minutos para conferir consolidação satisfatória aos painéis, devido ao aumento do teor de águas nas camadas superficiais do colchão de partículas.

Nascimento [12] estudou a produção em laboratório de chapas de partículas homogêneas com madeira da região da Caatinga do Nordeste Brasileiro: Angico (Anedananthera macrocarpa), Algaroba (Prosopis juliflora) e Jurema Preta (Mimosa tenuiflora).

Os procedimentos experimentais foram definidos em função das propriedades físicas e mecânicas das espécies estudadas, das características do adesivo utilizado, e das características das partículas utilizadas para confecção das chapas.

As chapas foram confeccionadas com partículas de aproximadamente 1,8 $\mathrm{mm}$ de comprimento, $10 \%$ de ureia-formaldeído com $5 \%$ de solvente (base peso seco), tempo de prensagem de dez minutos com temperatura entre 100 a $120^{\circ} \mathrm{C}$ e pressão de prensagem de $4 \mathrm{MPa}$.

Nessas condições, as propriedades físicas e mecânicas das chapas foram satisfatórias. Os valores médios de resistências apresentaram potencial equivalente às chapas fabricadas em escala industrial, possibilitando concluir que é viável a fabricação de chapas de partículas homogêneas de boa qualidade, com partículas de madeiras da Caatinga do Nordeste Brasileiro.

Análises realizadas por Boonstra [6] no pré-tratamento de partículas de madeira com vapor de água com temperaturas entre 200 a $210^{\circ} \mathrm{C}$, para produção de chapas de partículas, permitiram concluir que esse tipo de tratamento possibilita maior estabilidade dimensional às chapas, bem como influencia na melhoria de suas propriedades físicas e mecânicas.

\section{Resina Poliuretana à base de óleo de mamona}

Há uma tendência mundial na procura de materiais biodegradáveis, não poluentes e derivados de biomassa que, segundo Araújo [2], permitiu aumento nas pesquisas com poliuretanos derivados de óleo de mamona, ampliando as perspectivas para sua utilização.

O desenvolvimento dos poliuretanos derivados de óleo de mamona teve origem nos 
primeiros trabalhos propostos na década de 1940 [15]. O óleo de mamona é extraído da semente da planta Ricinus communis, que é encontrada em regiões tropicais e subtropicais, sendo muito abundante no Brasil. É um líquido viscoso, obtido pela compressão das sementes ou por extração com solvente.

Na Universidade de São Paulo, no Instituto de Química de São Carlos, desenvolveu-se o primeiro adesivo poliuretano derivado do óleo de mamona, do tipo bi-componente.

A partir deste recurso natural e renovável é possível sintetizar polióis e prepolímeros com diferentes propriedades que, quando misturados, dão origem a um poliuretano. Possuem grande versatilidade de aplicação com propriedades altamente interessantes [10].

$O$ cultivo da mamona vem sendo amplamente incentivado em alguns estados, como Mato Grosso, com o projeto Promamona/MT, Minas Gerais, com o Pró-Mamona, Paraíba e Bahia, o maior produtor brasileiro de mamona, entre outros.

Segundo o site do projeto Pró-Mamona, o plantio da mamona apresenta grandes vantagens ao agricultor: ela tem ciclo curto, entre 180 e 210 dias; o custo de produção gira em torno de R \$ 700,00 por hectare, menor que o custo do milho; a mamona, além disso, pode ser plantada em consórcio com outras culturas ou solteira (quando plantada sem associação a outras culturas, o teor de óleo contido em cada planta é de quase $50 \%)$; por fim, seu cultivo requer pouco uso de produtos químicos e possibilita grande facilidade na colheita, já que não há necessidade de máquinas e é resistente à seca.

Além dos benefícios ao agricultor, esta planta apresenta vantagens ao meio ambiente:

") Redução na emissão de dióxido de carbono $\left(\mathrm{CO}_{2}\right)$;

"Sequestro de $\mathrm{CO}_{2}$ da atmosfera, pois uma lavoura de um hectare de mamona pode absorver até oito toneladas de gás carbônico da atmosfera;

" Redução de emissão de óxidos de enxofre e hidrocarbonetos;

" Alta biodegradabilidade se comparada com óleo diesel mineral;

" Tecnologia limpa e utilização de subprodutos da cadeia produtiva;

") Reduz a emissão de materiais particulados (fuligem e fumaça preta).

Além de tudo, o emprego da resina poliuretana à base de mamona em substituição a resina de ureia-formaldeído elimina a indesejável emanação de formaldeído.

\section{Conclusões da revisão bibliográfica}

Em linhas gerais, pode-se afirmar que a revisão bibliográfica possibilitou a compreensão do tema em estudo e evidenciou a falta de informações bibliográficas referentes ao emprego de podas de árvores urbanas como insumos para a produção de painéis de partículas de madeira. 


\section{MATERIAIS E MÉTODOS}

\section{Materiais}

Para a realização deste trabalho, foram utilizados os seguintes materiais:

" Podas de árvores urbanas, da espécie Canelinha (Nectandra lanceolata). Estas espécies foram identificadas pelo Prof. Dr. Eduardo Chahud, FUMEC, Belo Horizonte, utilizando avaliação anatômica, de acordo com os métodos usualmente utilizados para tal tipo de análise;

" Resina poliuretana à base de óleo de mamona (bicomponente), constituída de pré-polímero e poliol;

" Luvas, máscaras e outros acessórios indispensáveis para atividades em laboratório.

\section{Métodos}

\section{Fabricação das chapas}

A madeira das duas espécies estudadas passou por um processo de separação das folhas (que não foram utilizadas no processo de produção), picagem em moinho específico e peneiramento para retirada do pó. Então, todo o material retido na peneira de malha 2,8 $\mathrm{mm}$ foi misturado com a resina e encaminhado para prensa hidráulica com capacidade para fabricar placas em escala de laboratório, com dimensões de $40 \mathrm{~cm} \mathrm{x} 40 \mathrm{~cm}$.

Para fabricar cada placa, foram utilizados $1500 \mathrm{~g}$ de cavaco aglutinado com $240 \mathrm{~g}$ de adesivo, totalizando $16 \%$ da massa de cavaco, contabilizando neste valor eventuais perdas no manuseio da mistura. Estes parâmetros de processo foram retirados de trabalhos desenvolvidos por Nascimento [12] e Dias [7].

Nesta fase, o material ainda permanece sem adesão, com aparência semelhante a uma farinha. Em seguida é colocado em um molde e recebe uma pequena pressão para que ganhe coesão e possa ser colocado na prensa hidráulica, à pressão da ordem de 3,5 $\mathrm{MPa}$, à temperatura de $90^{\circ} \mathrm{C}$.

Com este procedimento, procurou-se produzir as chapas de modo a representar adequadamente as etapas de fabricação industrial, ilustradas na Figura 1 


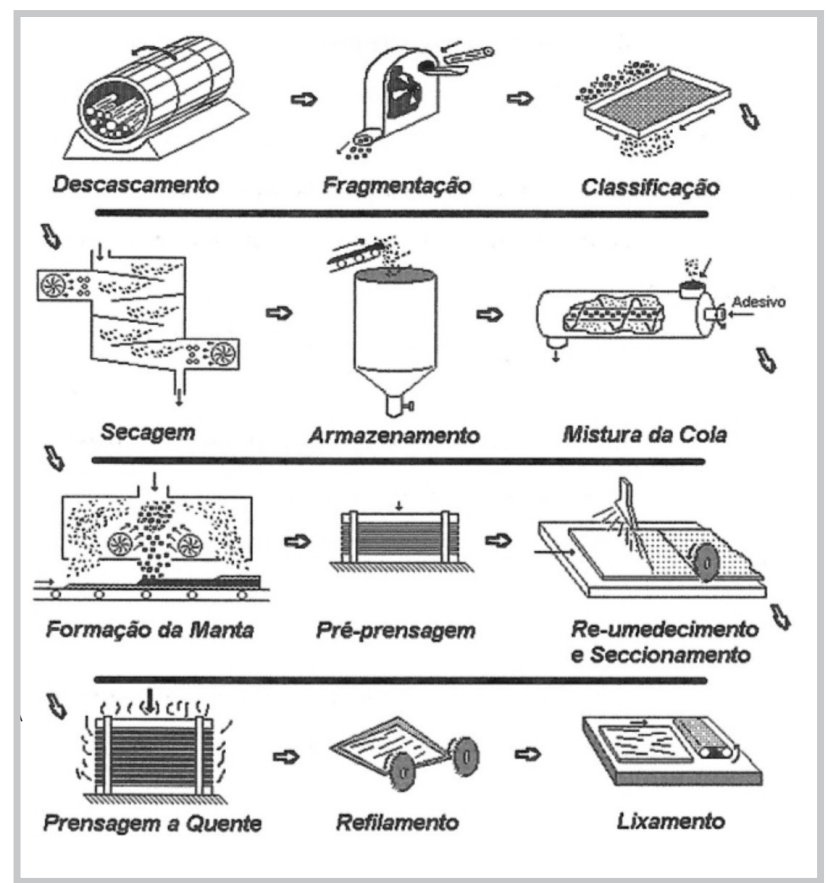

Figura 1 - Etapas de

fabricação das chapas de aglomerado (Particuladas)

Detalhes da produção em laboratório apresentam-se nas figuras a seguir.
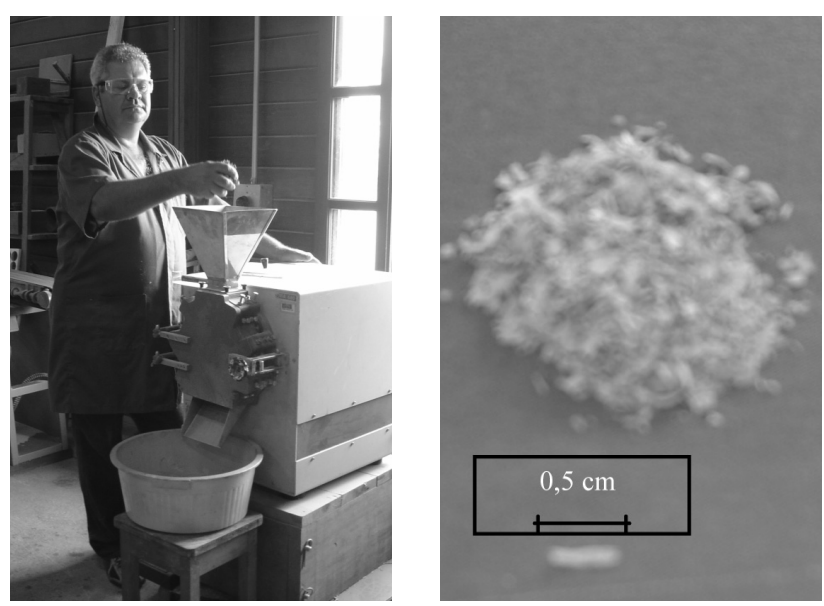

Figura 2 (à esquerda) - Moinho de faca para a obtenção das partículas

Figura 3 (à direita) - Partículas de Canelinha 
Figura 4 - Da esquerda para direita: Formadora de colchão, Colchão de partículas, Prensa hidráulica
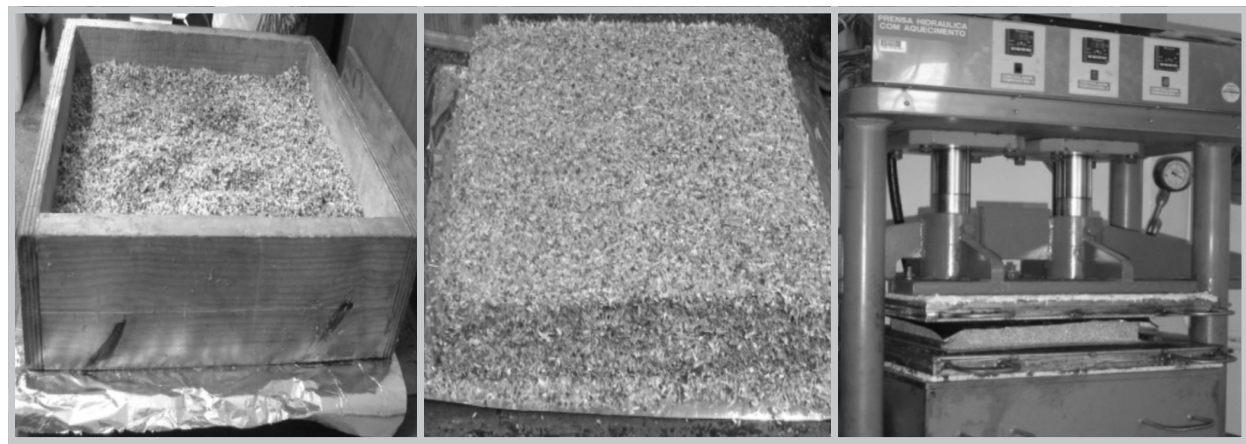

\section{Avaliação do desempenho das chapas}

Para análise da viabilidade de produção das chapas a partir dos insumos citados, foram realizados ensaios físicos e mecânicos com base na ABNT NBR 14810:2006, que prescreve no mínimo dez corpos de prova de cada propriedade por chapa comercial. No presente estudo, a quantidade foi reduzida de modo proporcional às dimensões das chapas produzidas $(40 \mathrm{~cm}$ x $40 \mathrm{~cm})$. Foram determinadas: densidade, umidade, resistência à tração perpendicular às faces/TP (adesão interna), módulo de resistência (MOR) e módulo de elasticidade (MOE) na flexão.

Registra-se que foram fabricadas seis chapas de cada uma das espécies mencionadas e seis chapas com partículas das duas espécies (50\% de cada uma delas). Foi adotada a seguinte notação:

" Chapas de Canelinha: identificadas pela letra inicial C, seguida do numeral correspondente a cada chapa da qual se retiram os corpos de prova para ensaios;

\section{RESULTADOS E DISCUSSÃO}

\section{Propriedades físicas das chapas produzidas}

Na Tabela 1 constam os valores médios das propriedades físicas.

Tabela 1 Densidade, teor de umidade, inchamento e absorção de água para chapas de partículas de Canelinha

\begin{tabular}{lcccc}
\hline CHAPA & $\begin{array}{c}\text { DENSIDADE P } \\
\left(\mathrm{KG} / \mathrm{M}^{3}\right)\end{array}$ & $\begin{array}{c}\text { UMIDADE U } \\
(\%)\end{array}$ & $\begin{array}{c}\text { INCHAMENTO } \\
(\%)\end{array}$ & $\begin{array}{c}\text { ABSORÇÃO } \\
(\%)\end{array}$ \\
\hline $\mathrm{CHC} 1$ & 853 & 5,7 & 3,4 & 7,4 \\
\hline $\mathrm{CHC} 2$ & 842 & 5,6 & 5 & 6,2 \\
\hline $\mathrm{CHC} 3$ & 867 & 5,6 & 4,9 & 8,5 \\
\hline
\end{tabular}




\begin{tabular}{|c|c|c|c|c|}
\hline CHAPA & $\begin{array}{c}\text { DENSIDADE P } \\
\left(\mathrm{KG} / \mathrm{M}^{3}\right)\end{array}$ & $\begin{array}{l}\text { UMIDADE U } \\
(\%)\end{array}$ & $\begin{array}{l}\text { INCHAMENTO } \\
(\%) 2 \text { HORAS }\end{array}$ & $\begin{array}{l}\text { ABSORÇÃO } \\
(\%) 2 \text { HORAS }\end{array}$ \\
\hline $\mathrm{CH} 44$ & 863 & 5,4 & 6,1 & 7,5 \\
\hline $\mathrm{CH} 55$ & 860 & 5,5 & 4,3 & 7,1 \\
\hline $\mathrm{CHC} 6$ & 849 & 5,2 & 4,2 & 6,1 \\
\hline MÉDIA & 856 & 5,5 & 4,7 & 7,1 \\
\hline D. PADRÃO & 10 & 0,18 & 0,9 & 0,9 \\
\hline${ }^{\star} \mathrm{CV}$ & 0,01 & 0,03 & 0,2 & 0,13 \\
\hline
\end{tabular}

*CV - Coeficiente de variação

De acordo com os requisitos da NBR 14810:2006, as chapas têm que apresentar densidade mínima compreendida entre $551 \mathrm{~kg} / \mathrm{m}^{3}$ e $750 \mathrm{~kg} / \mathrm{m}^{3}$. As chapas foram produzidas para densidade entre $856 \mathrm{~kg} / \mathrm{m}^{3}$ e $969 \mathrm{~kg} / \mathrm{m}^{3}$, ultrapassando requisitos da norma e confirmando resultados apresentados por Nascimento [12].

A NBR 14810:2006 especifica que o teor de umidade médio não pode ser inferior a $5 \%$ nem superior a $11 \%$, o que foi atendido por todas as chapas.

Quanto ao inchamento, o documento normativo apresenta $8 \%$ como valor máximo após imersão em água por duas horas. Todas as chapas produzidas atenderam a estas exigências. Cabe destacar que a NBR 14810:2006 não indica requisitos mínimos para absorção de água, o que impossibilita a comparação com os resultados aqui obtidos, embora sejam consistentes com os apresentados por Nascimento [12] e Dias [7].

Os valores do coeficiente de variação obtidos (no máximo 0,20) estão de acordo com aqueles obtidos pelos pesquisadores Nascimento e Dias, o que confere confiabilidade à consistência do processo de produção de painéis empregado no laboratório.

\section{Propriedades físicas das chapas produzidas}

Na Tabela 2 constam os valores médios das propriedades mecânicas.

Tabela 2 Densidade, teor de umidade, inchamento e absorção de água para chapas de partículas de Canelinha

\begin{tabular}{lccc}
\hline & & \multicolumn{2}{c}{ FLEXÃO ESTÁTICA (MPA) } \\
\cline { 3 - 4 } CHAPA & TP (MPA) & MOR & MOE \\
\hline CHC1 & 0,89 & 16,5 & 1656 \\
\hline CH C2 & 0,81 & 14,9 & 1589 \\
\hline CH C3 & 0,84 & 15,8 & 1674 \\
\hline
\end{tabular}




\begin{tabular}{lccc}
\hline \multirow{2}{*}{ CHAPA } & TP (MPA) & \multicolumn{2}{c}{ FLEXÃO ESTÁTICA (MPA) } \\
\cline { 3 - 4 } & & MOR & MOE \\
\hline CHC5 & 0,76 & 15,2 & 1602 \\
\hline CHC6 & 0,81 & 16 & 1545 \\
\hline MÉDIA & 0,8 & 15,9 & 1642 \\
\hline D. PADRÃO & 0,06 & 0,7 & 84 \\
\hline${ }^{*}$ CV & 0,08 & 0,04 & 0,05 \\
\hline
\end{tabular}

*CV - Coeficiente de variação

Para espessura variando de 8 a 13 mm, a NBR 14810:2006 especifica o valor mínimo de o,40 MPa para tração perpendicular. Todas as chapas apresentaram valores muito superiores ao requerido pela norma, mostrando o bom comportamento do adesivo utilizado.

A NBR 14810:2002 exige MOR mínimo de $18 \mathrm{MPa}$ para chapas com espessura compreendida entre $8 \mathrm{~mm}$ e $13 \mathrm{~mm}$, porém não é indicado valor mínimo de MOE na flexão estática. Embora nenhuma chapa tenha alcançado o valor exigido, pode-se perceber que é pequena a diferença entre a média de MOR obtido para as chapas e o requisito normativo. Fica evidente que é possível a obtenção de chapas que atendam a este requisito, variando a quantidade de adesivo ou parâmetros de processo (intensidade de pressão e temperatura, por exemplo), como já argumentaram Nascimento [12] e Dias [7].

\section{CONCLUSÕES}

No geral, os resultados obtidos no desenvolvimento deste trabalho mostraram a viabilidade técnica da produção de painéis particulados de madeira a partir de resíduos de podas de árvore urbana.

Diversos dos requisitos normativos foram alcançados com os parâmetros de processo utilizados. Os resultados específicos para o módulo de resistência na flexão estática (MOR) não atingiram os requisitos da NBR 14810:2006, embora os valores médios tivessem sido muito próximos a $18 \mathrm{MPa}$, como prescreve o referido documento.

Em trabalhos futuros será necessário ajustar os parâmetros de processo, como já apontado, para que os requisitos normativos sejam atingidos e viabilizem a proposição de uso dos painéis assim produzidos.

\section{REFERÊNCIAS BIBLIOGRÁFICAS}

[1] ALBUQUERQUE, C. E. C.; IWAKIRI, S. Interações de variáveis no ciclo de prensagem de aglomerados. Tese de doutorado, Curitiba: Universidade Federal do Paraná, 2002.

[2] ARAÚJO, L. C. R. Caracterização química e mecânica de poliuretanas 
elastoméricas baseadas em materiais oleoquímicos. Dissertação de mestrado, São Carlos: Instituto de Física e Química de São Carlos (USP), 1992.

[3] BLANCHET, P.; CLOUTIER, A.; RIEDL, B. Particleboard made from hammer milled black spruce bark residues. Wood Science and Technology, vol. 34, n. 1, pp. 11-19, 2000.

[4] BNDES. Painéis de madeira no Brasil: panorama e perspectivas. Banco Nacional de Desenvolvimento Econômico e Social. Setorial, n. 27, pp. 121-156, Rio de Janeiro, 2008. Disponível em http://www.bndes.gov.br/. Acesso em: 9 ago. 2009.

[5] BODIG, J.; JAYNE, B. A. Mechanics of wood and wood composites: characteristics of wood composites. Nova York: Van Nostrand Reinhold Company, 1982.

[6] BOONSTRA, M.J. The effects of a two stage heat treatment process on the properties of particleboard. Holz Roh-Und Werkstoff, vol. 64, n. 2, pp. 157-164, 2006.

[7] DIAS, F. M. Aplicação de resina poliuretana à base de mamona na fabricação de painéis de madeira aglomerada. Produtos Derivados da Madeira: síntese dos trabalhos desenvolvidos no Laboratório de Madeiras e de Estruturas de Madeira, SET-EESC-USP. São Carlos: Escola de Engenharia de São Carlos (USP), 2008, pp. 73-92.

[8] IWAKIRI, S. Painéis de Madeira Reconstituída. Curitiba: Fundação de Pesquisas Florestais - FUPEF, Universidade Federal do Paraná, 2005.

[9] IWAKIRI, S.; CRUZ, C. R.; OLANDOSKI, M. A. B. Utilização de resíduos de serraria na produção de chapas de madeira aglomerada de Eucalyptus saligna, Eucalyptus citriodora e Eucalyptus pilularis. Revista Floresta e Ambiente, v. 7, n. 1, pp. 251-256, 2000.

[10] JESUS, J. M. H. Estudo do adesivo poliuretano à base de mamona em madeira laminada colada (MLC). Tese de doutorado, São Carlos: Escola de Engenharia de São Carlos (USP), 2000.

[11] MENDES, M. L. Pinus spp na produção de painéis de partículas orientadas (OSB). Tese de doutorado, Curitiba: Universidade Federal do Paraná, 2001.

[12] NASCIMENTO, M. F. CPH - Chapas de Partículas Homogêneas: madeiras do nordeste do Brasil. Tese de doutorado, São Carlos: Escola de Engenharia de São Carlos (USP), 2003.

[13] POLETO, S. F. S.; NASCIMENTO, M. F.; MORALES, E. A. M.; ROCCO LAHR, F. A. Produção de chapas de partículas homogêneas utilizando resíduos de espécies de reflorestamento. In: Chahud, Eduardo. Reciclagem de Resíduos para a Construção Civil. Belo Horizonte: FUMEC-FEA, 2007.

[14] TOMASELLI, I. Tendências de Mudanças na Indústria de Painéis. Revista da Madeira, 2000.

[15] VILAR, W. D. Química e tecnologia dos poliuretanos. [S.I.]: Grupo Pronor, 1993.

\section{AGRADECIMENTOS}

A bolsista agradece à Pró-Reitoria de Cultura e Extensão da Universidade de São Paulo pela bolsa concedida no âmbito do Programa Aprender com Cultura e Extensão. 
EDUARDO CHAHUD professor doutor da Faculdade de Engenharia e Arquitetura da Universidade FUMEC, Belo Horizonte.

FRANCISCO ANTONIO ROCCO LAHR professor titular da Escola de Engenharia de São Carlos da Universidade de São Paulo.

KAREN ANÉRIS BLECHA bolsista do Programa Aprender com Cultura e Extensão da Pró-Reitoria de Cultura e Extensão Universitária da Universidade de São Paulo (PRCEU-USP).

MARIA FÁTIMA DO NASCIMENTO pós-doutoranda na Escola de Engenharia de São Carlos da Universidade de São Paulo - e-mail: frocco@sc.usp.br.

MARÍLIA DA SILVA BERTOLINI doutoranda no Programa Interunidades Ciência e Engenharia de Materiais da Universidade de São Paulo. 